\title{
Depression and Sensitization to Stressors Among Young Women as a Function of Childhood Adversity
}

\author{
Constance Hammen and Risha Henry \\ University of California, Los Angeles
}

\author{
Shannon E. Daley \\ University of Southern California
}

\begin{abstract}
The authors tested a stress-sensitization version of a diathesis-stress approach to depression. In a 2-year longitudinal follow-up design, exposure to stressful life events was examined in young women in the transition to adulthood. The authors hypothesized that those who had experienced one or more significant childhood adversities would have a lower threshold for developing a depressive reaction to stressors. Results indicated that women with exposure to one or more childhood adversities-such as family violence, parent psychopathology or alcoholism, and others-were more likely to become depressed following less total stress than women without such adversity. The results could not be accounted for by chronic stress or prior depression. Both biological and psychological sensitization mechanisms may be speculated to play a role, but the actual mechanisms of stress sensitization remain to be explored.
\end{abstract}

Diathesis-stress models of psychopathology have been embraced by most researchers who attempt to understand depressive disorders. The past two decades have witnessed extensive research on the psychological factors that constitute diatheses, or predispositions, and on methods for assessing stress and evaluating its role in depression. There has been somewhat less research that actually integrates these themes in diathesis-stress approaches testing onsets of depression over a longitudinal course. Yet the studies that do attempt such tests have generally demonstrated that the combination of high susceptibility (such as dysfunctional cognitions) and high stress predicts depression (e.g., Brown, Bifulco, \& Harris, 1987; reviews in Barnett \& Gotlib, 1988; Haaga, Ernst, \& Dyck, 1991; Nietzel \& Harris, 1990; see also Monroe \& Simons, 1991).

There are several limitations of the tests of diathesis-stress models of depression, however. One is that they have rarely examined the original Zubin and Spring (1977) hypothesis that different levels of diathesis may require different levels of stress. Monroe and Simons (1991) noted that most diathesis-stress models fail to consider different types of "interaction." Most studies test the model by examining an interaction term comprised of the multiplicative factors of stress and diathesis, and the resulting significant-interaction term supporting the diathesis-stress model is usually not specifically evaluated, or it takes the form that high diathesis plus high stress produces higher levels of depression than other combinations. An alternative is that high levels of diathesis may require low levels of stress, but this prediction has rarely been

Constance Hammen and Risha Henry, Department of Psychology, University of California, Los Angeles; Shannon E. Daley, Department of Psychology, University of Southern California.

This research was supported by an award from the William T. Grant Foundation. We are grateful to Dorli Burge, Joanne Davila, David Herzberg, and Nangel Lindberg, who served as interviewers.

Correspondence concerning this article should be addressed to Constance Hammen, Department of Psychology, 405 Hilgard Avenue, Los Angeles, California 90095. Electronic mail may be sent to hammen@ psych.ucla.edu. tested. A second limitation of tests of diathesis-stress models is related: The model is generally static and fails to consider that the threshold for stress-precipitation of reactions may change over the course of the disorder. Indeed, the distinct boundaries between diathesis and stress may be blurred to the extent that stress may change the diatheses by creating biological and cognitive changes that lower the threshold of responding to later stressors. An approach that takes account of a potentially dynamic process is especially important for developmental psychopathology models of depression (and other psychopathology). Such approaches attempt to understand the influence of earlier experiences on the developing organism and view disorder as the unfolding of a transaction between the person and the environment (e.g., Cicchetti \& Schneider-Rosen, 1986; Rutter, 1986). A developmental psychopathology approach thus might suggest that early-occurring stressors alter the threshold of stress needed to precipitate depressive reactions in susceptible individuals.

A third limitation of diathesis-stress tests of depression is that they have tended to assume that psychological diatheses such as maladaptive cognitions are acquired in childhood through experiences with the family environment but have rarely fleshed out and tested such ideas. Although there are important and intriguing exceptions to this relative neglect of childhood origins of dysfunctional cognitions (e.g., Garber, Weiss, \& Shanley, 1993; NolenHoeksema, Girgus, \& Seligman, 1992), considerable further work is needed to include and test the role of childhood experiences in the acquisition of susceptibility to depression.

A fourth limitation of diathesis-stress studies of depression is that they have made relatively scant progress in the potential integration of psychological and biological approaches to the diatheses for depression. Cognitive and biological models have often proceeded in isolation. Several characteristics of clinically significant depression have emerged in recent years, however, that seem to call out for integrative approaches. Among them are the finding that depression runs in families, that it is typically a recurrent disorder, and that it often first emerges in childhood or adolescence (e.g., Burke, Burke, Regier, \& Rae, 1990; Downey \& Coyne, 
1990; Hammen, 1991; Hammen, 1997; Hammen \& Rudolph, 1996).

Although it is beyond the scope of the current article to address all of these limitations, our purpose is to test a hypothesis that draws from these considerations. We propose to demonstrate a stress-sensitization effect in which individuals at risk show depressive reactions to lower levels of stress than do those not at risk. We hypothesize that childhood exposure to significant stress will be associated with a lower stressor threshold for depressive reactions. Thus, although we expect that depression will be associated with higher levels of prior stress than nondepression, among individuals who have experienced a depressive reaction we predict that those who were exposed to early childhood adversities will have lower stress levels preceding the depression than those who did not experience early childhood adversities.

The hypothesized mechanism draws conceptually on two recent approaches-biological psychiatry and sociology-as well as from cognitive vulnerability models of depression. Gold, Goodwin, and Chrousos (1988) postulated that some forms of depression arise from defects in biological stress mechanisms that may have been sensitized by exposure to acute childhood stressors; Post (1992) proposed that stressors and episodes of mood disorder cause neurobiological changes such that less stress is required to precipitate subsequent episodes. Although the current study does not itself test biological factors, it may be viewed as testing an approach compatible with a neurobiological process in which previous experiences alter the organism and affect the threshold at which stressors precipitate depression. Kessler and Magee (1993) demonstrated in a large epidemiological survey that exposure to childhood adversities was a vulnerability factor for development of initial as well as recurrent episodes of depression. It may be speculated that the empirical link between early adversity and later depression may be accounted for by the acquisition of both cognitive and biological vulnerabilities (diatheses), such that less stress is needed to precipitate depressive reactions.

The present study tested a stress-sensitization model of depressive reactions in a design uniquely suited for an initial investigation of this process in a naturalistic setting. Participants were all female, relatively homogeneous in age, socioeconomic status (SES), and exposure to life transitions, and they were followed over a longitudinal period with careful assessment of depressive experiences, stressful life events, and exposure to certain childhood adversities. The participants were young women in the transition between high school graduation and early adulthood-a period of significant risk for depressive experiences (e.g., Burke et al., 1990; Lewinsohn, Hops, Roberts, Seeley, \& Andrews, 1993) as well as challenging life experiences. On the basis of the scale reported by Kessler and Magee (1993), we assessed the occurrence of several adverse experiences in childhood, adolescence, or both: serious family mental health problems, severe alcohol problems, exposure to violence in the family, death of a parent, parental marital conflict, parental divorce, and lack of a confiding relationship with an adult. Most of these have been identified as risk factors for depressive and other disorders (e.g., reviewed in Hammen \& Rudolph, 1996).

We hypothesized that although prior stress would be higher in general among those who developed depressive reactions than those who did not, individuals with a history of exposure to these adversities will develop a depressive reaction (major or minor depressive episode) when exposed to a lower amount of stress in comparison with women who did not have such backgrounds. Stress was measured in terms of exposure in the 3 months prior to depression onset (or in a randomly selected 3-month period for those who did not become depressed). We further hypothesized that the findings would not be explained by differences in prior history of depression or chronic ongoing stressful conditions including family relationships. The latter controls for the possibility that it is high levels of overall chronic stress or, specifically, high levels of ongoing family adversity, rather than sensitization to lower episodic stress, that could account for depression onset.

\section{Method}

\section{Participants}

Recruitment. Participants were recruited from the senior classes of three local high schools that were chosen to be demographically representative of Los Angeles County high schools in terms of ethnicity, proportion graduating, and proportion attending college after graduation (Los Angeles County Office of Education, 1991). Students were administered brief questionnaire packets in their homeroom classes after giving consent to participate. The goal was to obtain female volunteers for a longitudinal interview study who represented a cross-section in terms of demographic characteristics, symptoms, and post-high school activities. Recruitment was done in two cohorts, one during 1991 and the other during 1992. Out of 513 who gave consent (or if not yet 18, whose parents gave consent) and completed the initial questionnaires during the senior year, 341 indicated willingness to participate in the interview and follow-up if contacted. However, when the time came several months later shortly after high school graduation, only 155 could actually be located and scheduled. Some had moved or were traveling; some declined when they further considered that the follow-up was asking for multiple years of participation; some simply could not be located; some were scheduled but did not show up or had transportation difficulty that made coming to the University of California, Los Angeles (UCLA) for the interviews prohibitive; and finally, some were working and simply could not be scheduled. To evaluate representativeness of the final interview sample, we conducted two sets of comparisons on the initial questionnaire and demographic variables: (a) the 341 initial volunteers (who stated during screening that they would be willing to be contacted for further interview participation) versus the 172 initial nonvolunteers (who completed the questionnaires but did not wish to be in the interview follow-up study); and (b) among the 341 initial volunteers, the 155 participants versus the 186 who subsequently declined or could not be scheduled. There were no significant differences among volunteers and nonvolunteers, or those who were and were not eventually interviewed in terms of SES (measured by the guidelines of Hollingshead, 1975), ethnicity, self-reported depressive symptoms, or number of behavior problems endorsed on the screening measure. Among the 155 in the interview study, there were no significant differences between the two cohorts interviewed 1 year apart, 94 in the first cohort and 61 in the second cohort, and they were thus combined for all analyses. Thus, because of the school selection and the lack of differences between all who were screened and those who actually participated, we believe that the sample is representative of senior women students who were attending classes on the day of the initial testing.

Characteristics. The 155 women who began the study entered in the summer after high school graduation, with a mean age of 18.29 $(S D=0.48)$ years. The self-reported ethnicity of the sample was as follows: African American 2\%; Asian American 9\%; Caucasian 46\%; Chicano/Latino $21 \%$; Other (mostly mixed-race or Armenian descent) $22 \%$. The mean Hollingshead socioeconomic score of $46.03(S D=13.18)$ indicates that the sample was predominantly middle and lower middle class.

The present study drew from 121 women who completed the first 2 years of the follow-up and who also completed the questionnaire packets at 
Year 3 or 4 that included the adversity checklist. Additionally, one woman with bipolar disorder was excluded, and one was excluded because she had a depression onset within 5 days of the initial interview, and therefore life stress data for a 3-month period were not available. A comparison of the 121 with the 34 who were not included in the present analyses indicated no significant differences on history of prior depression or initial depression at the beginning of the study.

\section{Procedures}

During the summer following high school graduation, each woman participated in an initial face-to-face interview. Follow-up interviews were conducted at 6 months and then annually, largely by phone, with questionnaires being sent by mail. The present study covers the first 2 years of follow-up. All interviews were conducted by male and female graduate students in clinical psychology or by licensed clinical psychologists. Interviewers were trained to proficiency on the Structured Clinical Interview for DSM-III-R (SCID) and life stress procedures, and subsequent reliability analyses were conducted (described later). Procedures including measures not relevant to the current study are reported in Hammen et al. (1995) and Daley et al. (1997). Interviewers were unaware of the hypotheses of the present study and were unaware of participants' history of experience with adverse childhood events.

Depression. Current and lifetime depressive disorders and symptomatology and other diagnostic conditions were assessed at each interview using the Structured Clinical Interview for DSM-III-R, developed for nonclinical samples (SCID-NP; Spitzer, Williams, Gibbon, \& First, 1992). The reliability and validity of the SCID for diagnoses covered are comparable with those of other commonly used diagnostic schedules (Williams et al., 1992). Interrater reliability of SCID diagnoses was assessed in the present study on the basis of independent ratings of SCID interviews made from audiotapes, computed as weighted kappas. For the initial interview $(n=46)$, reliability of current diagnostic status was $\mathrm{K}_{w}=.89$; for follow-up interviews $(n=20)$ it was $\mathbf{K}_{w}=.93$.

In order to have an operational definition of depression onset (significant change in depression level), SCID-based depression diagnoses were transformed into a 5-point scale indicating severity of symptoms or diagnoses: 0 (no depressive symptoms), 1 (one or two mild symptoms), 2 (three or more symptoms: minor depressive episode or nearly meets criteria for major depression), 3 (diagnosed major depressive episode or dysthymia), and 4 (major depressive episode plus serious suicide attempt or hospitalization). The SCID-based scales cover depressive symptomatology for the current month at the initial interview; for the follow-ups they cover the entire period between interviews. The correlations between SCID scaled values and concurrent Beck Depression Inventory (BDI) scores at the 6-month, 1-year, and 2-year assessments were $r=.51, r=.50$, and $r=.36$ (all $p$ s $<$ $.0001)$. Values were possibly attenuated as a result of slightly different timeframes (e.g., BDI was mailed and self-administered a week or two following the interviews). As noted below, a 2-point change in SCID-based depression level was used as the definition of onset in the analyses to be reported.

Stressful life events. At the initial evaluation and each subsequent follow-up, participants were administered a life event interview. The episodic life stress interview was modeled after the contextual threat assessment methods of Brown and colleagues (e.g., Brown \& Harris, 1978) and has been used extensively by Hammen and colleagues (e.g., Hammen, 1991; Hammen, Ellicott, Gitlin, \& Jamison, 1989). Self-report ratings and questionnaire checklists have been criticized because they may either fail to characterize the unique meaning of an event for an individual or elicit distorted perceptions of the significance of an event because depressed individuals might erroneously attribute causal significance to an event because of "effort after meaning." This term was used by Brown and Harris (1978) to describe the possible exaggeration of the importance of a negative event by the sufferer in order to explain their depression. Therefore, the goal of the interview approach is to obtain sufficient information about the surrounding circumstances of an event's occurrence to characterize as objectively as possible its impact on the person's life. The interviewer inquires about various areas of the person's life, identifying and carefully dating events that have occurred in the past period (in the present study, since the previous interview). Each reported event is probed to obtain information about what happened, how long it continued, whether it was expected, whether the person had ever experienced similar events, what resources and supports were available to the person, what the consequences were, and any other relevant background. Narrative accounts of each event containing this information were then prepared by the interviewer. Each report is presented to an independent rating team, omitting any information about the individual's actual reaction to the event. Consistent with the contextual threat methods of Brown and Harris, the team rates how much impact an event would have for a typical person under the same conditions. An objective impact, or threat rating, of each event is assigned on a scale ranging from 1 (no negative impact) to 5 (extremely severe negative impact).

Reliability of severity ratings was obtained by comparing two separate teams' scores. Intraclass correlations for events from a randomly chosen set of participants were .92 for objective impact ( $n=74$ events). Unlike Brown and Harris (1978), we did not require that an event have long-term impact in order to be scored.

For the present study, the primary stress variable was total objective stress score for the relevant 3-month interval prior to depression onset and was generated by adding the objective impact ratings for all events (excluding those with an impact of 1 , i.e., not at all stressful). The mean total stress score for the sample was $4.12(S D=3.90)$. Also, presence and number of severe negative events during the 3-month period prior to depression onset was determined on the basis of events assigned an objective impact rating above moderate (which is 3 on our 5-point scale) Mean number of severe life events was $0.22(S D=.54)$. For nondepressed individuals, a random 3-month period during the 2 years was selected for counting total stress and presence of severe stressors.

Timing of depression onset and prior stress. Timelines were created that charted depressive symptomatology and life events for each participant over the course of the first 2 years of the study. Participants were considered to have had a significant onset or exacerbation of depressive symptomatology if they experienced a change of two or more points on the five-point SCID-based depression scale $(n=48 ; 27$ major depressive episode, 21 minor depressive episode). For those participants with onsets or exacerbations, we tabulated the number of severe events occurring in the 3-month period preceding the onset or exacerbation. This methodology allowed us to ensure that episodic stress measured actually preceded depression and likely precipitated it in participants who became depressed, rather than being a correlate or consequence. For all other participants $(n=$ 73) a 3-month period was randomly chosen. Where participants had multiple episodes, the first episode was used. The use of the 3-month time frame is the convention in our research program (e.g., Hammen et al., 1995), and we believe it to be conceptually consistent with a cognitivebehavioral mechanism in which interpretations of events and their consequences elicit negative affect and eventuates in somatic, behavioral, $\operatorname{cog}$ nitive, and emotional elements of the depression syndrome.

Chronic stress. Chronic stress in several domains (work, school, finances, close friendship, family relationships, social life, romantic relationship) was assessed at each follow-up using an interview format developed for prior studies (e.g., Hammen et al., 1987). Using standard probes, each area is discussed covering the period since the previous interview, and each domain is rated on a five-point scale. Each scale has behaviorally specific points, ranging from 1 (exceptionally good circumstances) to 5 (extremely stressful and maladaptive). Intraclass correlations for independent judges based on 57 participants in the present study ranged from .82 to .91 . Other reliability and validity information is reported in Hammen et al. (1987). For the present analyses a chronic stress index was computed by summing across all domains.

Childhood adversity. Participants completed an adaptation of the Kessler and Magee (1993) Questionnaire of Early Childhood Adversities at 
the Year 3 (Cohort 2) or Year 4 (Cohort 1) follow-up. Kessler and Magee assessed seven types of childhood adversity, each focusing on an experience before the respondent was 16 years of age. They included (a) serious parental drinking problems, (b) serious parental mental health problem, (c) parental marital problems, (d) parental divorce or separation, (e) death of parent, (f) absence of a close and confiding relationship with parent or some adult, $(\mathrm{g})$ experience or witness violence in the family.

Reliability analyses were performed on the childhood adversity questionnaire by readministering the questionnaire at a later follow-up. The kappas were as follows: .80 for serious parental drinking problem, .76 for parental mental health problem, .92 for death of parent, .66 for experiencing or witnessing violence in the family, .83 for parent divorce or separation, .77 for parent marital conflict. The item "absence of a close and confiding relationship with parent or some adult" had $K<.20$ and so was excluded from analyses. The reliabilities were diminished because of an inadvertent slightly different time frame indicated on the two questionnaires administered at different follow-ups. The great majority of all the events were reported to have occurred between birth and age 5 (with the exception of parental death), and most were reported to have continued for long periods during the woman's younger life. Mean number of childhood adversities reported was $1.41(S D=1.45)$.

\section{Results}

The dependent variable was dichotomous: presence or absence of depression onset or exacerbation following the selected life event interval ( $n=73$ no onset; $n$ s $=27$ major depressive episodes and 21 minor depressive episodes as previously described).

Separate logistic regression analyses were conducted first to determine the predictive relationship (odds ratios [OR]) of stress and adversity to depression onset. The OR values indicate that for a one unit increase in the independent variable, the risk of depression is multiplied by the value of the OR. Both the main effects of total objective stress $(\mathrm{OR}=1.21 ; 95 \%$ confidence interval $[\mathrm{CI}]$ $=1.08-1.35)$ and the number of severe life events $(O R=4.37$; 95\% CI $=1.72-11.11$ ) were highly significant predictors of depression onset, $p<.0002$. There was a nonsignificant trend for childhood adversity by itself to predict depression onset $(\mathrm{OR}=1.27)$. The zero-order correlation between childhood adversities and the primary stress variable, total objective stress, was $.14(n s)$.

Logistic regression analyses were next conducted to examine the combined contributions of childhood adversity, total objective stress, and their interaction to depression onset. To control for the potentially confounding influence of chronic stress and history of prior depression (presence or absence of prior episodes), we entered these variables in the first step. In the second step we entered childhood adversity and total objective stress. As the critical test of the sensitization hypothesis, the third step contained the interaction between childhood adversity and total objective stress. Table 1 summarizes the analyses. The control variables of the first step were nonsignificant, and the second step containing total stress and adversity was highly significant. Of particular interest to the sensitization hypothesis, after controlling for the previous variables, the interaction between childhood adversity and total objective stress significantly predicted depression onset or exacerbation, $\chi^{2}(1, N=121)=9.42, p<.002 .^{1}$

To illustrate the direction of the interaction effect graphically, we dichotomized the independent variables - childhood adversity and total objective stress-on the basis of median split. Next, the dichotomized variables and their interaction were entered into the logistic regression, as in previous analyses. Using the final regres-
Table 1

Logistic Regression Analysis Predicting Exacerbation or Onset of Depression From Childhood Adversity and Life Stress

\begin{tabular}{lrrcc}
\hline \multicolumn{1}{c}{ Variable } & $\beta$ & $S E$ & $\begin{array}{c}\text { Odds ratio } \\
(95 \% \mathrm{CI})\end{array}$ & $\begin{array}{c}\text { Change in } \\
\chi^{2} \text { for step }\end{array}$ \\
\hline 1. Chronic stress & -.84 & .42 & $0.43(0.19-0.98)$ & \\
$\quad$ Past depression & .22 & .42 & $1.25(0.54-2.85)$ & $5.57(n s)$ \\
2. Childhood adversity & .17 & .15 & $1.18(0.89-1.57)$ & \\
$\quad$ Total objective stress & .17 & .06 & $1.18(1.05-1.33)$ & $10.46^{*}$ \\
3. Adversity $\times$ Stress & -.15 & .06 & $0.86(0.77-0.96)$ & $9.42^{*}$ \\
\hline
\end{tabular}

Note. $\quad N=121 . \mathrm{CI}=$ confidence interval.

$* p<.005$, two-tailed.

sion equation, we then calculated the mean predicted probability of onset or exacerbation for each of the four cells. As indicated in Figure 1, the pattern suggests that under high stress conditions, individuals have an increased likelihood of depression regardless of adverse childhood experiences, whereas under low stress conditions, those with more adversity exposure are more likely to become depressed $(p=.045)$.

\section{Discussion}

The goal of the study was to test a possible sensitization effect in which depressive reactions follow lower levels of stressors among those who were exposed to significant childhood adversity. The findings demonstrated the hypothesized stress-sensitization effect and support a stress-sensitization mechanism approach to the diathesis-stress model of depression in which presence of the diathesis (consequences of early exposure to adversity) lowers the threshold for the amount of stress preceding a depressive reaction. Results of this longitudinal study of young women demonstrated that those with exposure to one or more childhood adversitiessuch as family violence, parent psychopathology or alcoholism, death of a parent, or parental discord or divorce-were more likely to become depressed following less total stress than women without such early adversity. The effects for total stress were not accounted for by differences in previous depressive experiences-a process that might also alter the organism and affect neurobiological sensitivity to stress (e.g., Post, 1992). Nor were they explained by current chronic stressful conditions, because chronic stress prior to onset was statistically controlled. Although it is possible that early adversity also predicts continuing stress through the later years, control for current chronic stress suggests that the apparent sensitization effect is not due merely to continuing adversity.

\footnotetext{
${ }^{1}$ We also examined onset or exacerbation of depressive symptoms as a function of severe life events (e.g., Brown \& Harris, 1978). Logistic regression analyses were conducted controlling for prior depression and chronic stress at the first step, childhood adversity and number of severe life events at the second step, and the interaction of the two latter variables at the last step. The control variables were nonsignificant predictors of depressive episode, and the adversity and severe event variables at Step 2 were significant predictors, $\chi^{2}(2, N=121)=12.84, p=.0016$. At the third step the interaction of adversity and number of severe life events was marginally significant, $\chi^{2}(1, N=121)=3.06, p=.08$, two-tailed, but showed the same pattern of results as represented in Figure 1.
} 


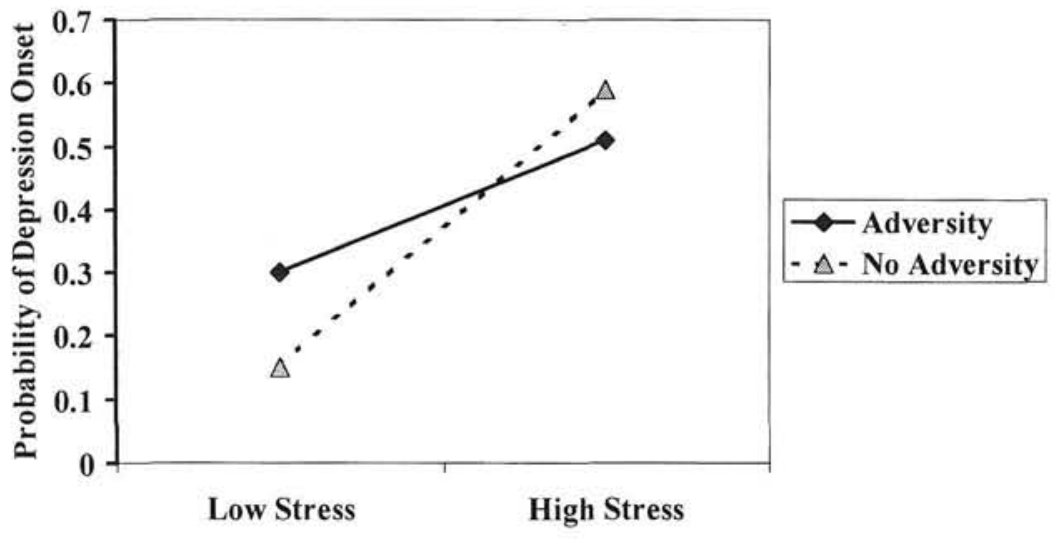

Figure 1. Probability of depression onset as function of stress and childhood adversity.

The novel findings of the present study require replication and further exploration. We acknowledge that this study does not demonstrate the precise mechanisms of stress sensitization. We can only speculate at this point that psychological and biological factors affect the threshold at which stress precipitates depression. However, the findings are consistent with a developmental psychopathology model of depression in which one pathway to vulnerability is through adverse family conditions, and such conditions may function through both cognitive and neurobiological mechanisms to affect future susceptibility to depression in the face of stress (e.g., Cicchetti \& Schneider-Rosen, 1986). Thus, a sensitization mechanism blurs the distinction between stress and diathesis, suggesting that stress (in this case, childhood adversity) creates a diathesis to react depressively in the face of future stressors, perhaps operating by neurobiological and psychological (cognitive) mechanisms. Cognitive theorists have long hypothesized-although rarely tested-a model of cognitive vulnerability to depression through the acquisition of maladaptive schemas that arise in childhood and are presumably based on adverse experiences in the family (e.g., Beck, Rush, Shaw, \& Emery, 1979; Nolen-Hoeksema, Girgus, \& Seligman, 1992). Segal, Williams, Teasdale, and Gemar (1996) speculated about a possible cognitive sensitization effect in which increasing accessibility of negative schemas results from repetition and frequent usage. By extension, long-practiced negative ways of viewing the self and the world may be triggered by minor events, resulting in depressive reactions due to negative information processing.

There are also intriguing models of vulnerability that focus on neurochemical changes to the organism as a consequence of exposure to stressors. In humans, alterations of hypothalamicpituitary-adrenal (HPA) axis functioning have been speculated to contribute to depression, and such processes may involve early stress exposure that sensitizes specific neural circuits resulting in depressive reactions later in life in response to stressful life events (e.g., Gold et al., 1988; Plotsky, Owens, \& Nemeroff, 1998). Most of the relevant research has been conducted on animals, but a growing body of human research has shown abnormal HPA axis functioning associated with adverse childhood experiences such as insecure attachment and child abuse (e.g., Gunnar, 1998; Heim, Ehlert, Hanker, \& Hellhammer, 1998). It is also possible that genetic factors may directly contribute to stress reactivity. Considerable further work is required to characterize the nature of the diatheses acquired in exposure to adversity.
In addition to conceptual gaps, it is also acknowledged that in the absence of appropriate comparison groups of nondepressive psychopathology, it cannot be claimed that this approach is specific to depressive disorders. Further limitations of the study include the homogeneity of the sample, limited to young women of the same age who were high school graduates and relatively homogenous in SES - although of diverse ethnic composition. Thus, results may not be generalizable to other segments of the population, including young men. Although there is no particular expectation that the genders would differ, it remains to be demonstrated that the current effects apply to men. The sample consisted of volunteers rather than randomly selected participants, possibly introducing bias of unknown characteristics. However, the sample reflected the demographic characteristics of local high school senior women, and the volunteer and nonvolunteer participants in the initial screening did not differ on demographic or psychosocial variables. Also, a high rate of lifetime major depression was observed in this sample (although not substantially higher than the younger adolescent sample observed by Lewinsohn and colleagues in Lane County, Oregon; Lewinsohn et al., 1993). Whether this indicates that our sample was at risk for depression in ways not readily detected when we first recruited them into the study during their high school senior years is not presently known. Further, we acknowledge reliance on self-reports of childhood adversity. With respect to the latter, however, if there are inaccuracies in women's reports, they are likely to be failure to report events that actually occurred rather than the reverse. Although interview methods of obtaining historical information about adversity would be desirable, the Kessler-Magee instrument that we added to the study had adequate reliability. Also, it is highly probable that there are additional childhood adversities that affect individuals that were not studied, as well as personal themes and contents that make certain types of events more likely to precipitate depression than others (such as interpersonal losses or conflicts). Although negative events occurring in early life were the focus of the study, we cannot rule out exposure of adverse events occurring later in life that might also have affected sensitivity to stressors. Sample size precluded study of the relative impact of individual adversities and whether the number or combination is important. Sample size also precluded limiting the study to only those with major depressive episodes, although we would argue that even minor episodes of depression represent significant im- 
pairment and maladaptation (cf. Gotlib, Lewinsohn, \& Seeley, 1995; Johnson, Weissman, \& Klerman, 1992).

Despite these limitations, the study had several strengths-use of objective scoring of stressors, SCID-based depression diagnoses, a longitudinal design, and a participant population at demographic risk for depression (young women) - all of which support a sensitization effect that could not be explained by psychiatric or chronic stress factors. Further research on individual differences in thresholds for depressive reactions to stress may help to clarify the continuing puzzle of heterogeneity of depressive experiences and may help to elucidate the mechanisms responsible for depression in the face of stress among those exposed to adverse childhood experiences.

\section{References}

Barnett, P. A., \& Gotlib, I. H. (1988). Psychosocial functioning and depression: Distinguishing among antecedents, concomitants, and consequences. Psychological Bulletin, 104, 97-126.

Beck, A. T., Rush, A. J., Shaw, B., \& Emery, G. (1979). Cognitive therapy of depression. New York: Guilford Press.

Brown, G. W., Bifulco, A., \& Harris, T. O. (1987). Life events, vulnerability and onset of depression: Some refinements. British Journal of Psychiatry, 150, 30-42.

Brown, G. W., \& Harris, T. (1978). Social origins of depression. London: Free Press.

Burke, K. C., Burke, J. D., Regier, D. A., \& Rae, D. S. (1990). Age at onset of selected mental disorders in five community populations. Archives of General Psychiatry, 47, 511-518.

Cicchetti, D., \& Schneider-Rosen, K. (1986). An organizational approach to childhood depression. In M. Rutter, C. E. Izard, \& P. E. Read (Eds.), Depression in young people (pp. 71-134). New York: Guilford Press.

Daley, S., Hammen, C., Burge, D., Davila, J., Paley, B., Lindberg, N., \& Herzberg, D. (1997). Predictors of the generation of episodic stress: A longitudinal study of late adolescent women. Journal of Abnormal Psychology, 106, 251-259.

Downey, G., \& Coyne, J. C. (1990). Children of depressed parents: An integrative review. Psychological Bulletin, 108, 50-76.

Garber, J., Weiss, B., \& Shanley, N. (1993). Cognitions, depressive symptoms, and development in adolescents. Journal of Abnormal Psychology, 102, 47-57.

Gold, P. W., Goodwin, F. K., \& Chrousos, G. P. (1988). Clinical and biochemical manifestations of depression: Relation to the neurobiology of stress. The New England Journal of Medicine, 319, 348-419.

Gotlib, I., Lewinsohn, P., \& Seeley, J. (1995). Symptoms versus a diagnosis of depression: Differences in psychosocial functioning. Journal of Consulting and Clinical Psychology, 65, 90-100.

Gunnar, M. R. (1998). Quality of early care and buffering of neuroendocrine stress reactions: Potential effects on the developing human brain. Preventive Medicine, 27, 208-211.

Haaga, D. A., Ernst, D., \& Dyck, M. J. (1991). Empirical status of cognitive theory of depression. Psychological Bulletin, 110, 215-236.

Hammen, C. (1991). Depression runs in families: The social context of risk and resilience in children of depressed mothers. New York: SpringerVerlag.

Hammen, C. (1997). Depression. London: Psychology Press.

Hammen, C., Adrian, C., Gordon, D., Burge, D., Jaenicke, C., \& Hiroto, D. (1987). Children of depressed mothers: Maternal strain and symptom predictors of dysfunction. Journal of Abnormal Psychology, 96, 190198.

Hammen, C., Burge, D., Daley, S., Davila, J., Paley, B., \& Rudolph, K. (1995). Interpersonal attachment cognitions and prediction of symptom- atic responses to interpersonal stress. Journal of Abnormal Psychology, 104, 436-443.

Hammen, C., Ellicott, A., Gitlin, M., \& Jamison, K. (1989). Sociotropy/ autonomy and vulnerability to specific life events in patients with unipolar depression and bipolar disorders. Journal of Abnormal Psychology, 98, 154-160.

Hammen, C., \& Rudolph, K. (1996). Childhood depression. In E. J. Mash \& R. A. Barkley (Eds.), Child psychopathology (pp. 153-195). New York: Guilford Press.

Heim, C., Ehlert, U., Hanker, J. P., \& Hellhammer, D. H. (1998). Abuserelated post-traumatic stress disorder and alterations of the hypothalamic-pituitary-adrenal axis in women with chronic pelvic pain. Psychosomatic Medicine, 60, 309-318.

Hollingshead, A. B. (1965). Four factor index of social status. Yale University, New Haven, CT: Author.

Johnson, J., Weissman, M., \& Klerman, G. (1992). Service utilization and social morbidity associated with depressive symptoms in the community. Journal of the American Medical Association, 267, 1478-1483.

Kessler, R., \& Magee, W. (1993). Childhood adversities and adult depression: Basic patterns of association in a U.S. national survey. Psychological Medicine, 23, 679-690.

Lewinsohn, P. M., Hops, H., Roberts, R. E., Seeley, J. R., \& Andrews, J. A. (1993). Adolescent psychopathology: I. Prevalence and incidence of depression and other $D S M-I I I-R$ disorders in high school students. Journal of Abnormal Psychology, 102, 133-144.

Los Angeles County Office of Education. (1991). The condition of public education in Los Angeles County 1990-1991 [annual statistical review]. Los Angeles: Author.

Monroe, S. M., \& Simons, A. D. (1991). Diathesis-stress theories in the context of life stress research: Implications for the depressive disorders. Psychological Bulletin, 110, 406-425.

Nietzel, M. T., \& Harris, M. J. (1990). Relationship of dependency and achievement/autonomy to depression. Clinical Psychology Review, 10, 279-297.

Nolen-Hoeksema, S., Girgus, J. S., \& Seligman, M. E. P. (1992). Predictors and consequences of childhood depressive symptoms: A 5-year longitudinal study. Journal of Abnormal Psychology, 101, 405-422.

Plotsky, P. M., Owens, M. J., \& Nemeroff, C. B. (1998). Psychoneuroendocrinology of depression. Psychoneuroendocrinology, 21, 293-307.

Post, R. M. (1992). Transduction of psychosocial stress into the neurobiology of recurrent affective disorder. American Journal of Psychiatry, $149,999-1010$.

Rutter, M. (1986). The developmental psychopathology of depression: Issues and perspectives. In M. Rutter, C. E. Izard, \& P. B. Read (Eds.), Depression in young people: Clinical and developmental perspectives (pp. 3-30). New York: Guilford Press.

Segal, Z. V., Williams, J. M., Teasdale, J. D., \& Gemar, M. (1996). A cognitive science perspective on kindling and episode sensitization in recurrent affective disorder. Psychological Medicine, 26, 371-380.

Spitzer, R., Williams, J., Gibbon, M., \& First, M. (1992). The Structured Clinical Interview for $D S M-I I I-R$ (SCID). I. History, rationale, and description. Archives of General Psychiatry, 49, 624-629.

Williams, B., Gibbon, M., First, M., Spitzer, R., Davies, M., Borus, J., Howes, M., Kane, J., Pope, H., Rounsaville, B., \& Wittchen, H. (1992). The Structured Clinical Interview for DSM-III-R (SCID). II. Multisite test-retest reliability. Archives of General Psychiatry, 49, 630-636.

Zubin, J., \& Spring, B. (1977). Vulnerability-A new view of schizophrenia. Journal of Abnormal Psychology, 86, 103-126.

Received June 7, 1999

Revision received March 27, 2000 Accepted April 5, 2000 